\title{
A New Approach to Teaching Feedback
}

\section{Appealing to a nontraditional audience with an updated curriculum}

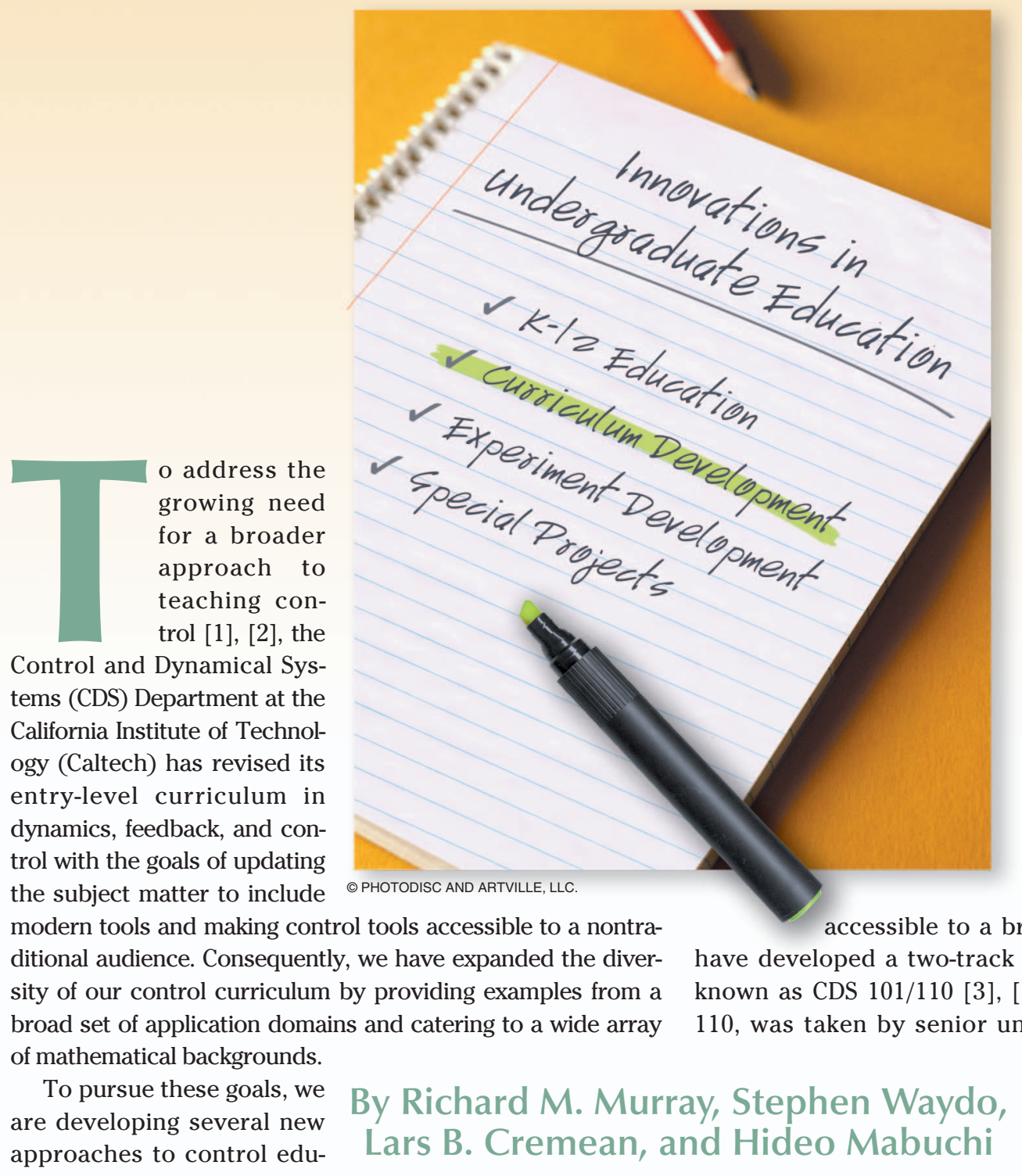

more detailed mathematical treatment of feedback. In this article we primarily discuss the conceptual track. The second approach is the development of a new undergraduate minor in control and dynamical systems.

\section{CDS 101/110: A Two-Track Approach to Introducing Control}

To make feedback concepts modern tools and making control tools accessible to a nontraditional audience. Consequently, we have expanded the diversity of our control curriculum by providing examples from a roader group of students, we have developed a two-track introductory control course known as CDS 101/110 [3], [4]. The original course, CDS 110 , was taken by senior undergraduates and first-year graduate students from all areas of engineering but was not suitable for students in scientific disciplines due to its heavy focus on engineercation, two of which are described here. The first approach is to divide the introductory control theory class into two tracks, with a conceptual track geared toward students who need only a conceptual overview of control tools and an analytical track providing a

ing examples. With increasing interest in control from other communities, the course was revised to provide an introduction to control concepts for students in biology, computer science, economics, environmental science, and 
physics. This revision led to the creation of a conceptual track, Design and Analysis of Feedback Systems (CDS 101), aimed at nontraditional students, and a revision of the analytical track, Introductory Control Theory (CDS 110), to include modern approaches and nonstandard examples from diverse application domains. In the fall of 2003, 72 students enrolled in CDS 101/110. Twelve students took the conceptual track, a number we expect will increase in the future as the course becomes better known across campus.

\section{CDS 101: Design and Analysis of Feedback Systems}

The conceptual track, CDS 101, is intended for advanced students in science and engineering who can benefit from an overview of control techniques but who might not have the need for the mathematical depth underlying the material. Special attention is paid to ensuring that the course is accessible to students from biological, physical, and information sciences, using examples from these domains to illustrate concepts. The goal of the course is to enable students to use the principles and tools of feedback in their research activities. In particular, after taking the course, students should be able to build control-oriented models of physical, biological, or information systems and simulate those systems in the time-domain; analyze stability, performance, and robustness of the models; and design rudimentary feedback control systems in the time and frequency domains. Special emphasis is given to state-space methods for analysis and synthesis, since these techniques are needed for systems that are nonlinear and asynchronous.

Lectures for CDS 101 consist of a one-hour conceptual lecture and a one-hour research lecture each week. The conceptual lectures, given on Monday, introduce the main ideas using PowerPoint presentations (see Figure 1) and assume minimal mathematical background. Hardware demonstrations are used to convey concepts whenever possible, along with simulations and interactive MATLAB sessions. Examples are drawn from relevant engineering topics such as cruise-control design for the Defense Advanced Research Projects Agency (DARPA) Grand Challenge vehicle (see sidebar, "The DARPA Grand Challenge") and from less traditional applications such as electrical power networks and predator-prey systems.

A second lecture, given on Friday, is used to explore research applications of control in different disciplines. Heavy emphasis is placed on nontraditional applications of feedback, with lectures given by Caltech faculty who are experts in those disciplines. Examples include insect flight (M. Dickinson), quantum feedback (H. Mabuchi), and Internet congestion control (S. Low).

The homework sets for CDS 101 consist of two problems each week, one of which is a computer exercise. The computer exercises use MATLAB and SIMULINK and involve problems of moderate complexity to demonstrate the power of the tools. The homework requires approximately four to six hours to complete, and typical problems include evaluating the stability of equilibrium points using phase-portrait analysis in MATLAB and creating and experimenting with control-oriented models, such as SIMULINK block diagrams.

\section{Integration with Existing Courses}

CDS 101 is integrated with the existing control course Introductory Control Theory (CDS 110). Students in CDS 110 are generally from a more conventional audience of junior and senior chemical, electrical, and mechanical engineers and have a stronger mathematical background than their CDS 101 counterparts. The emphasis here is on the standard set of control concepts such as linear ODEs, frequency-domain analysis, and design of feedback

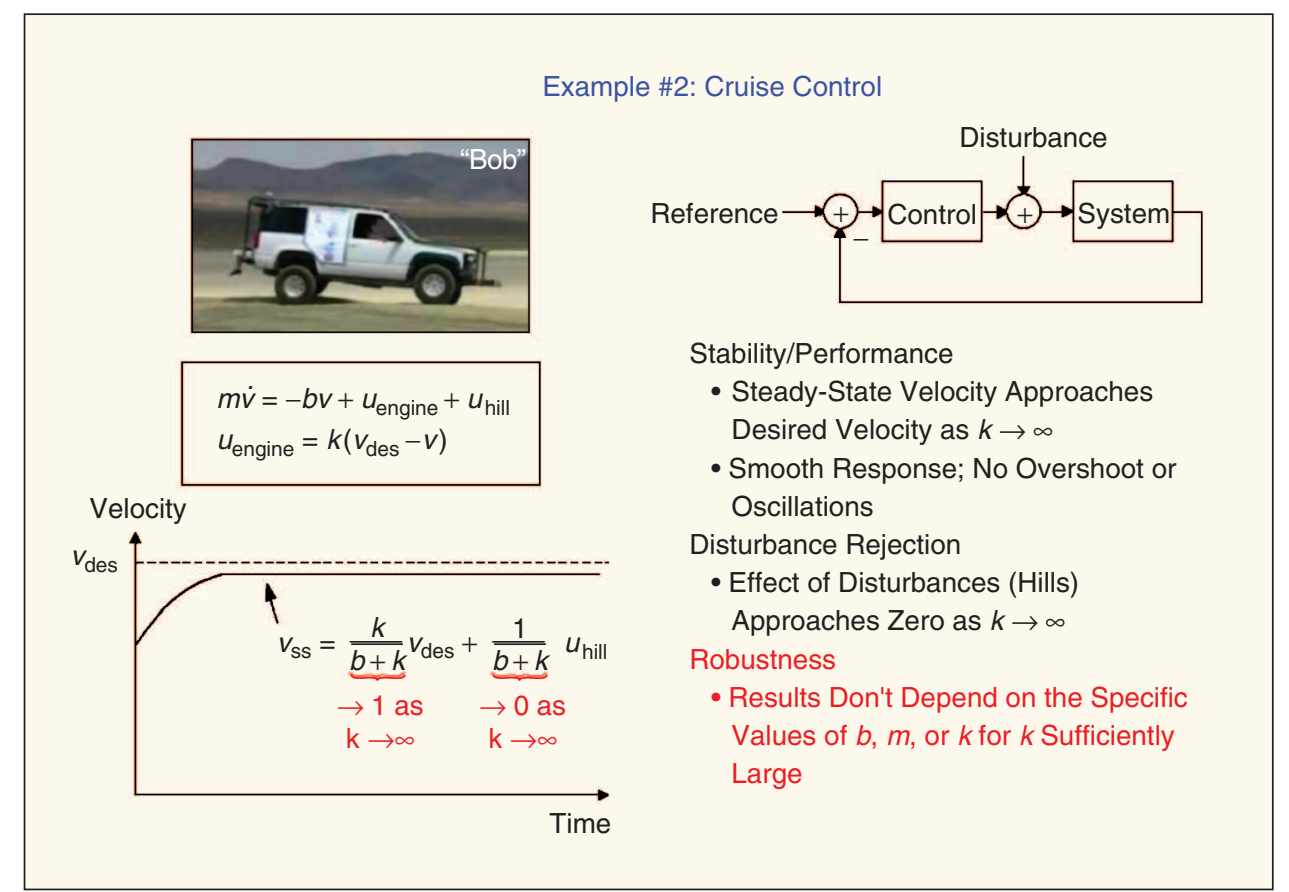

Figure 1. Example slide from a conceptual lecture. This lecture, which is the first of the term, is intended to motivate feedback control. Cruise-control design for the DARPA Grand Challenge vehicle is used as a real-world example. 


\section{The DARPA Grand Challenge}

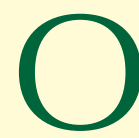

n 13 March 2004, Caltech participated in the

DARPA Grand Challenge [6], a race across the

Mojave desert for fully autonomous land vehicles. Caltech's team [7] was composed almost entirely of undergraduate students, several of whom were enrolled in CDS 101/110. This project was both a realistic, largescale engineering problem with a significant controls component for the students involved and a source of real-world examples for the CDS 101/110 lectures and homework. For example, the vehicle's speed controller was used throughout the term to demonstrate analysis and design techniques.

The preparation of Caltech's entry for the Grand Challenge began one year before the competition, with over 60 undergraduates participating. In the final three months before the race, student involvement was organized as a formal course for multidisciplinary project engineering dubbed DGC 120. This course was organized with several learning objectives that are particularly applicable to large-scale project engineering, including managing personal and project time, running effective meetings, organizing and prioritizing project tasks, and working in teams across traditional engineering boundaries.

DGC 120 provided an unprecedented opportunity for Caltech students to be engaged in a control engineering project in which the concepts and tools used in CDS 101/110 could be directly applied and where their use could be seen in the improved performance of a fullscale autonomous vehicle. This effort culminated in Caltech's vehicle being one of only seven to successfully navigate the 1.25-mile Qualification, Inspection, and Demonstration course, thereby becoming one of 15 vehicles to qualify for the race. In the actual race the vehicle drove 1.3 mile autonomously before becoming stuck on a barbed-wire fence, for the fifth-best showing. Caltech has begun preparations for the next Challenge, to be held in mid-2005, by improving our vehicle's performance while seeking more effective and creative ways to integrate the teaching of control theory and applications.

systems. Additionally, we provide an exposure to tools not usually taught at this level, such as controllability and observability analysis of state-space systems and Lyapunov stability analysis of nonlinear systems. The goal of this track is to build the same skills as in CDS 101 and provide the analytical background needed to derive and go beyond the results presented in the conceptual lectures.
In addition to attending the CDS 101 lectures and doing the same homework as CDS 101 students, CDS 110 students attend a second two-hour whiteboard lecture each week in which a more traditional mathematical approach is taken to deriving the results presented in the conceptual lectures. These lectures use tools from linear algebra, complex analysis, dynamical systems, and additional homework problems requiring these tools are assigned to students in this track. Typical homework problems are advanced versions of those given to CDS 101 students, including more design elements and more in-depth analysis. Feedback from students in CDS 110 has indicated that the dual conceptual plus technical lecture format has been helpful in enhancing understanding of the key concepts of the course.

\section{Pedagogy}

In addition to covering a somewhat unusual set of topics, CDS 101 integrates a number of nontraditional teaching methods to increase the effectiveness of the course.

\section{Mud Cards and FAQ Database}

Mud cards [5] are a simple tool for allowing students to get additional information on topics that they did not understand in the lecture. Blank $3 \times 5$ cards are handed out at the beginning of each lecture, and the students are instructed to write on the cards the muddiest part of the lecture. The cards are collected at the end of the class, and the teaching assistants (TAs) post responses on the Web by the evening of the lecture, rapidly providing information for students who had questions. The same online database is used to post responses to frequently asked questions on homework, so these answers are available to students as they work on assignments. Typical questions range from clarification of details in the lecture, to conceptual questions regarding the material, to administrative questions and comments about the course.

\section{Coinstructors}

CDS 101 is jointly taught by several faculty, including a main lecturer and three to four additional co-instructors. The co-instructors include biologists, physicists, and computer scientists, and thus represent the students in many of the areas that the course seeks to reach. The instructors are responsible for giving one to two lectures during the term, typically including one of the research lectures. All instructors and the head TA meet weekly to discuss the material that will be presented in the following week and to coordinate research lectures with the primary lectures. These meetings also provide an important mechanism for continuous improvement of the course material.

\section{Course Surveys}

We conduct a small survey three times over the term-at the first lecture, at the midterm exam, and at the final. The 
first survey focuses on identifying the mathematical background of students taking the course. Since this background varies from year to year, we use this data to adjust the content of lectures to supplement areas of weakness. The midterm and final surveys include these questions so we can evaluate progress, as well as an evaluation of what teaching tools have been most useful to students-conceptual lectures, detailed lectures, mud cards, office hours. We also leave room on the midterm and final surveys for comments on the course, which the students use heavily. Figure 2 summarizes survey results for Fall 2003.

\section{Undergraduate Minor in CDS}

In addition to updating our course offerings, we have introduced an undergraduate minor in CDS. The CDS minor is designed for students who wish to broaden their knowledge beyond their major or who may wish to pursue a graduate program involving control or dynamical systems. The minor was introduced in the 2003-04 academic year, and is already being pursued by several students.
The requirements for the CDS minor are modest, consisting of at least three courses in CDS, including one in control and one in dynamical systems, and carrying out a senior thesis project using CDS tools. All CDS courses applied to the CDS minor requirements must be taken for grades, and students must obtain a grade of B or higher. The senior thesis requirement can be satisfied by completing a three-term senior thesis in the student's major program using CDS subject matter, with the approval of the thesis topic by the CDS faculty, or by taking a separate course.

The goal of the CDS minor is similar to that of our curriculum reforms; namely, to broadly disseminate the principles and techniques developed in control beyond their traditional engineering audience. In fact, we are finding that many of the students interested in the minor come from traditional engineering backgrounds and seek to specialize in control through the minor. Since most engineering programs do not require a senior thesis, this participation has the secondary effect of increasing the students' undergraduate research activities.

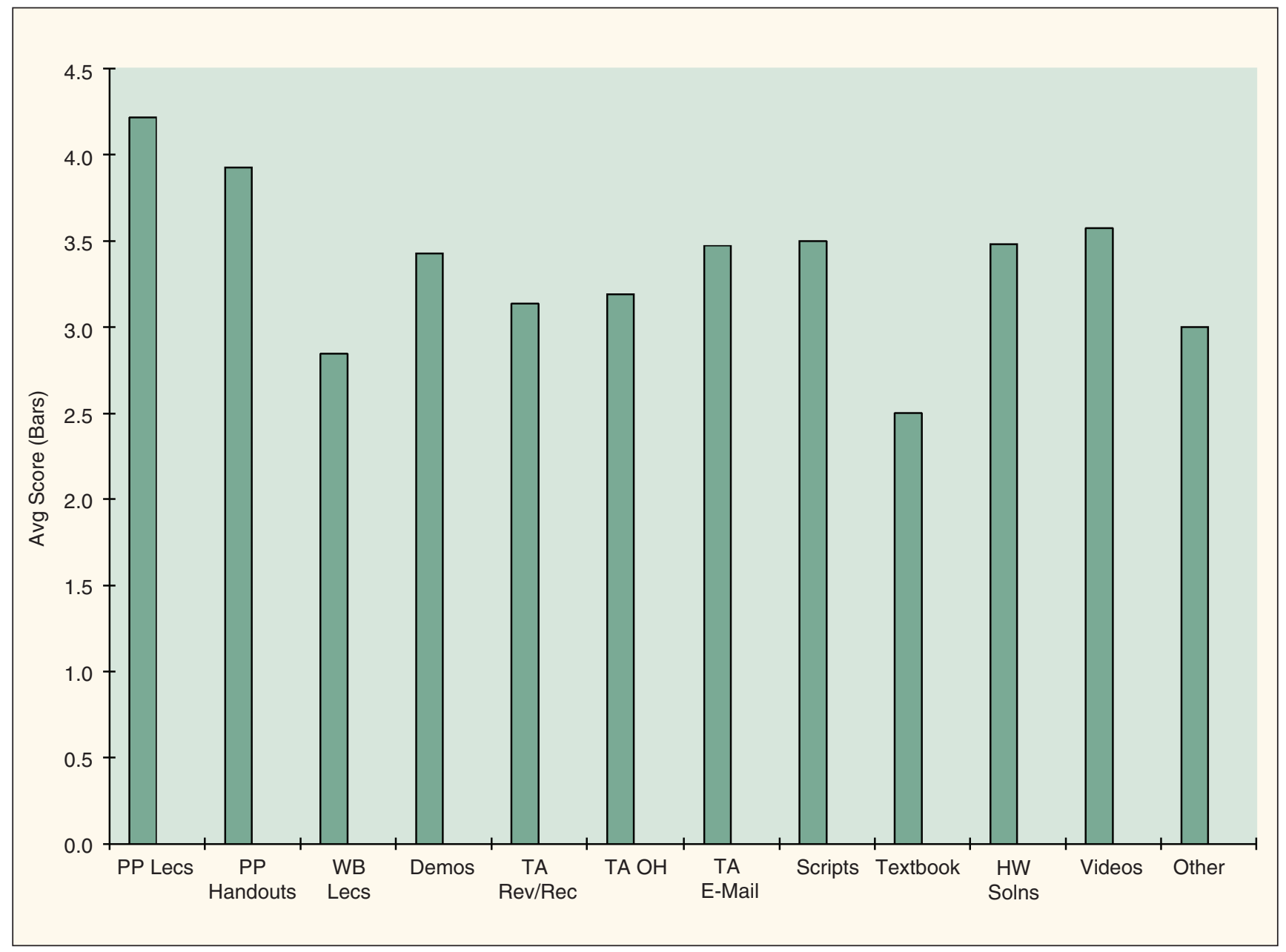

Figure 2. Results from the final survey for fall 2003. The survey shows that students preferred the PowerPoint (PP) lectures to the white board (WB) lectures, and that the textbook (an unpublished work in progress) was not satisfactory. 


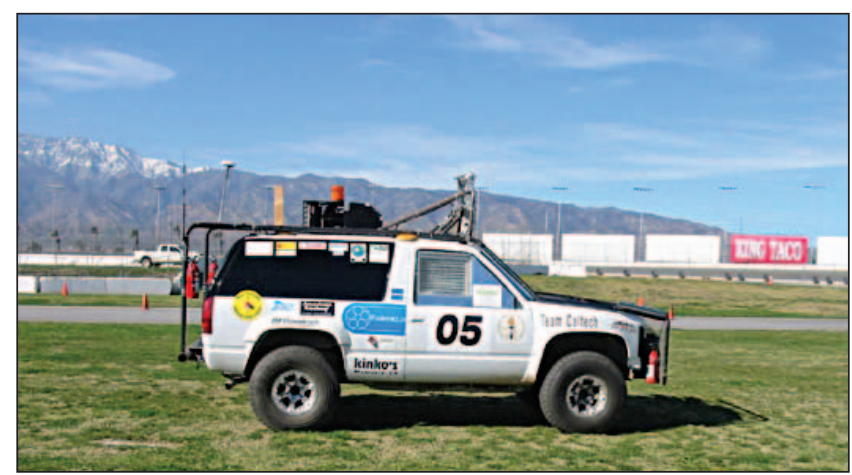

Figure 3. Team Caltech's DARPA Grand Challenge entry. This photo was taken at the Qualification, Inspection, and Demonstration test the week before the race.

While it is too early to tell whether the undergraduate minor will be an effective means for broadening the base of students interested in control, we are encouraged by the initial interest.

\section{Conclusions}

Through revised course offerings and new educational programs, the CDS Department at Caltech is seeking to expand the level of interest in control beyond its roots in aeronautical, chemical, electrical, and mechanical engineering into new fields such as biology, computer science, economics, environmental science, and physics. These changes are well suited to the environment at Caltech, which emphasizes science and mathematics, and which allows flexibility in the undergraduate curriculum.

In addition to these initial steps, we plan to add new courses that focus on system-level issues in engineering, such as those arising in the DARPA Grand Challenge. Interconnection and feedback are critical for understanding and designing complex systems, and new tools will be required to build and operate such systems reliably and efficiently. In future years, we hope to add a new course in multidisciplinary project engineering, as well as a freshman level course in engineering systems, which can increase students' interest in systems and control at an early stage in their education.

\section{References}

[1] R.M. Murray, Ed., Control in an Information Rich World. Philadelphia, PA: SIAM, 2003, pp. 71-80. Available: http://www.cds.caltech .edu/ murray /cdspanel.

[2] R.M. Murray, K.J. Åström, S.P. Boyd, R.W. Brockett, and G. Stein, "Future directions in control in an information-rich world," IEEE Contr. Syst. Mag., vol. 23, pp. 20-33, Apr. 2003.

[3] R.M. Murray, "Future directions in control, dynamics, and systems: Overview, grand challenges, and new courses," Eur. J. Control, vol. 9, no. 2, pp. 144-158, 2003.

[4] R.M. Murray. (2003). CDS 101/110 course Web page. [Online]. Available: http://www.cds.caltech.edu/ murray/courses/cds101/fa03/
[5] S. Hall, I. Waitz, D. Brodeur, D. Sodergolm, and R. Nasr, "Adoption of active learning in a lecture-based engineering class," in Proc. ASEE/IEEE Frontiers in Educ. Conf., Boston, MA, 2002, pp. T2A-9-15. Available: http://www.cdio.org/papers/papers.html

[6] (2004). DARPA Grand Challenge Web page. [Online]. Available: http://www.darpa.mil/grandchallenge/

[7] D. van Gogh. (2004). Team Caltech Web page. [Online]. Available: http://www.team.caltech.edu/

Richard M. Murray (murray@cds.caltech.edu) received his B.S. degree in electrical engineering from the California Institute of Technology in 1985 and his M.S. and Ph.D. degrees in electrical engineering and computer sciences from the University of California, Berkeley, in 1988 and 1991, respectively. He is currently a professor of mechanical engineering and control and dynamical systems and division chair of engineering and applied science at the California Institute of Technology, Pasadena. Prof. Murray's research is in the application of feedback and control to mechanical, information, and biological systems. He can be contacted at Control and Dynamical Systems 107-81, California Institute of Technology, 1200 E California Blvd., Pasadena, CA 01125 USA.

Stephen Waydo received his B.S. in aeronautics and astronautics from the University of Washington (UW) in 2001 and is now a Ph.D. candidate in control and dynamical systems at Caltech. His research interests include control of multiagent systems, particularly with respect to verification of algorithms distributed across multiple mobile vehicles. At Caltech he is supported by a Fannie and John Hertz Foundation Fellowship, and while at UW he was the recipient of a Mercury Seven Astronaut Scholarship.

Lars B. Cremean received his B.S. in mechanical engineering from Cornell University in 1999 and his M.S. in mechanical engineering from Caltech in 2000. He is currently a Ph.D. candidate in mechanical engineering with a minor in control and dynamical systems. His research is in cooperative control of multivehicle and robotic systems, and in the winter 2004 term he served as a teaching assistant for DGC 120 and manager of the planning team for Team Caltech's entry in the DARPA Grand Challenge.

Hideo Mabuchi received his A.B. from Princeton University and his Ph.D. from Caltech. He has worked in optical and atomic physics, using a combination of experimental and theoretical approaches. His research focuses on the use of realtime feedback for active control of quantum systems, nonequilibrium dynamics of biomolecular systems, and quantum information science. Dr. Mabuchi is currently an associate professor of physics and control and dynamical systems at the California Institute of Technology. Selected honors include an A. P. Sloan Research Fellowship and a John D. and Catherine T. MacArthur Foundation Fellowship. 